\title{
ENSINO PROFISSIONALIZANTE: UMA REFLEXÃO ACERCA DA FORMAÇÃO DE CUIDADORES DE IDOSOS
}

\author{
Luís Felipe Pissaia ${ }^{1}$ \\ Jéssica Maria Moccelin ${ }^{2}$ \\ Arlete Eli Kunz da Costa ${ }^{3}$ \\ Márcia Jussara Hepp Rehfeldt ${ }^{4}$ \\ Claudete Moreschi ${ }^{5}$
}

\begin{abstract}
Resumo: O envelhecimento populacional é destacado como um fenômeno mundial e, atrelado a este processo, observa-se uma elevação no número de doenças crônico-degenerativas. Consequentemente, estas mudanças geram a necessidade de cuidados permanentes, surgindo como apoio à formaçáo de cuidadores para aqueles que pretendem exercê-los. Por meio deste estudo objetiva-se realizar uma reflexão acerca do ensino profissionalizante na formação do cuidador de idosos. Utilizaram-se como bases para a reflexão diversas obras científicas que abordam a temática, além da experimentação em uma turma de cuidadores de idosos de uma escola profissionalizante do interior do Rio Grande do Sul, Brasil. Com base na reflexão proposta, considera-se que a profissionalização destes indivíduos torna-se necessária à medida que o contingente populacional idoso aumenta, a fim de garantir uma assistência qualificada e integral para esta população específica, além disso, identificou-se que práticas de ensino baseadas em metodologias de problematização fortalecem a apreensão do conhecimento e posterior contextualização sobre seu papel enquanto profissional.
\end{abstract}

Palavras-chave: Ensino. Cuidadores. Idosos.

1 Enfermeiro. Mestrando em Ensino. Univates. Lajeado - RS - Brasil. lpissaia@universo.univates.br

2 Acadêmica de Enfermagem. Univates. Lajeado - RS - Brasil. jessica.moccelin@universo.univates.br

3 Enfermeira. Doutora em Ambiente e Desenvolvimento. Univates. Lajeado - RS - Brasil. arlete.costa@univates.br

4 Doutora em Informática na Educação. Univates. Lajeado - RS - Brasil. mrehfeld@univates.br

5 Enfermeira. Doutora em Ambiente e Desenvolvimento. Univates. Lajeado - RS - Brasil. clau_moreschi@yahoo.com.br 


\title{
PROFESSIONAL EDUCATION: A REFLECTION ON THE TRAINING OF CAREGIVERS OF ELDERLY
}

\begin{abstract}
Population aging is highlighted as a worldwide phenomenon and linked to this process there is an increase in the number of chronic-degenerative diseases. Consequently, these changes generate the need for permanent care, appearing as support for the training of caregivers for those who intend to exercise them. Through this study we aim to carry out a reflection about vocational training in the formation of caregivers of the elderly. A number of scientific works dealing with this theme were used as a basis for reflection, as well as experimentation in a group of elderly caregivers from a vocational school in the interior of Rio Grande do Sul, Brazil. Based on the proposed reflection, it is considered that the professionalization of these individuals becomes necessary as the elderly population increases, in order to guarantee a qualified and integral assistance for this specific population, in addition, it was identified that teaching practices Based on methodologies of problematization strengthen the knowledge apprehension and later contextualization about its role as a professional.
\end{abstract}

Keywords: Teaching. Caregivers. Elderly.

\section{INTRODUÇÃO}

Atualmente quando se delimita uma análise sobre a população idosa brasileira torna-se perceptível seu gradativo aumento no contingente numérico da faixa etária, bem como diversas dificuldades atreladas ao desenvolvimento de uma velhice com qualidade principalmente com o apoio de uma assistência com qualidade (MIRANDA; MENDES; SILVA, 2016). Neste sentido, o objetivo deste estudo é traçar uma reflexão acerca do ensino profissionalizante na formação do cuidador de idosos (ROSA, 2016).

Justifica-se a necessidade deste estudo visto à expansão da demanda de cuidadores de idosos, bem como na constante modificação do perfil de saúde e doença dos indivíduos, bem como nas representações em que suas limitações são apresentadas (CAETANO; TAVARES, 2017). Para tanto quando se reflete sobre a formação destes profissionais, logo são relacionados com a qualidade de vida do idoso, principalmente no desenvolvimento e fortalecimento de suas habilidades (MIRANDA; MENDES; SILVA, 2016).

Em consonância com este determinante o cuidador de idosos detém em seu perfil profissional uma postura funcional que responde diretamente as necessidades desta população, seja pela bagagem cultural que cerca o meio, ou pelos novos modelos de ensino profissionalizante (ROSA, 2016). Mas em suma, as características de um cuidador de idosos perpassam as questões formais e políticas que gerenciam suas atribuições, pois denotam a construção de um perfil crítico e reflexivo pautado na necessidade de mudança e qualificação da assistência (CAETANO; TAVARES, 2017).

Buscando realizar a reflexão proposta elencaram-se quatro categorias temáticas que compreendem grandes áreas de representação, sendo a primeira intitulada "Envelhecimento social" onde se discute a evolução do envelhecimento 
humano na sociedade contemporânea e sua repercussão nos modelos comunitários. Já a segunda categoria "A vulnerabilidade social e necessidade de cuidados" perpassando por questões muito discutidas sobre as necessidades assistenciais do idoso e os principais tipos de vulnerabilidades que acometem esta população.

A terceira categoria temática "Perfil contemporâneo do cuidador de idosos" trata das discussões modernas sobre a profissionalização deste novo agente assistencial, traçando um limiar histórico sobre seu trabalho e disposição frente à legislação. Já a última categoria "Ensino transformador e formador" apresenta dados encontrados por meio da realização de uma aula reflexiva com alunos de um curso profissionalizante de cuidador de idosos, onde se buscou sensibilizar os futuros profissionais por meio da reflexão e autocrítica sobre o próprio trabalho.

\section{ENVELHECIMENTO SOCIAL}

Atualmente, o Brasil presencia um momento de transição demográfica, ou seja, um país que até então possuía uma população majoritariamente jovem, hoje esta envelhecendo, as constatações começaram a ser fundamentadas a partir do momento em que se evidenciou uma queda drástica nas taxas de fecundidade acompanhada pelo aumento da expectativa de vida (ROSA, 2016). Fato este relacionado com o desenvolvimento e expansão do mercado farmacêutico e inserção de novos modelos assistenciais que reverteram o perfil de saúde e doença presente até o século XIX, onde a predominância de óbitos ocorria por meio de doenças infectocontagiosas (LIMA; DELGADO, 2017).

Contudo, o aumento da expectativa de vida impulsionou um novo cenário de patologias que até então permaneciam desconhecidas, sendo elas as Doenças Crônicas Não Transmissíveis (DCNT), que acometem principalmente a população idosa, sendo responsável pelos casos de invalidez e óbito (AZEVEDO; GAZETTA; SALIMENE, 2016). Segundo estimativas nacionais, cerca de $85 \%$ da população idosa possui pelo menos uma DCNT após os 60 anos de idade, na maioria dos casos os sintomas ou até mesmo o diagnóstico ocorre anos antes, fato este de que os hábitos de vida contemporâneos aceleram seu desenvolvimento nas demais faixas etárias (MIRANDA; MENDES; SILVA, 2016).

Além do aparecimento das DCNT incluem-se nas taxas de vigilância em saúde as comorbidades ou agravos decorrentes do aparecimento inicial de uma doença crônica, representando cerca e 10\% do total de indivíduos diagnosticados (LIMA; DELGADO, 2017). Mesmo assim as DCNT não representam a totalidade de patologias enfrentadas pela população, estando à mercê de doenças degenerativas que se desenvolvem com o avanço da idade e independente dos avanços da medicina preventiva, constituindo-se principalmente nos casos de Doença de Alzheimer e Parkinson (PETRELLA; PINTO, 2016).

Estudos realizados a nível mundial atrelaram o aparecimento da Doença de Alzheimer ao aumento da idade do indivíduo, representando no mínimo 15\% dos diagnósticos realizados na população idosa mundial (MIRANDA; MENDES; SILVA, 2016). Ressalta-se ainda a vulnerabilidade que a faixa etária representa 
dentre os demais segmentos da sociedade, por presenciarem mudanças drásticas no ciclo biológico e fisiológico corporal, estando ligado a um processo de alteração nas percepções emocionais e interação social com a família e a comunidade (ROSA, 2016).

Outro fator de relevância neste contexto de envelhecimento populacional é a queda no rendimento financeiro familiar, ocasionado pela aposentadoria ou incapacidade de exercer atividades remuneradas, além de maiores gastos com tratamentos de doenças e de recursos pessoais de apoio à assistência (CAETANO; TAVARES, 2017). Torna-se fato que na terceira idade o aparecimento de doenças e a acentuação de incapacidades resultantes do processo de envelhecimento geram a necessidade de apoio de terceiros, sejam eles familiares, desconhecidos, profissionais da área da saúde ou que estejam capacitados para exercer tal função (PETRELLA; PINTO, 2016).

\section{A VULNERABILIDADE SOCIAL E NECESSIDADE DE CUIDADOS}

Quando atrelamos o envelhecimento populacional à vulnerabilidade a ela inferida, obtemos um contexto desdenhado em dificuldades amplamente constituídas em problemáticas que envolvem saúde, educação, recursos financeiros, além de uma ampla gama de fatores estressores que desempenham papel crucial nesta fase vital (AZEVEDO; GAZETTA; SALIMENE, 2016). Infere-se que a presença de diversos fatores torna a vulnerabilidade ainda mais exposta à realidade, pois os conjuntos de características que apresentam deficiência em seu desempenho acabam por desencadear alterações em dimensões ampliadas no modo de viver deste indivíduo (ROSA, 2016).

Em consonância a isso, Salmazo-Silva et al. (2012) apontam que o processo de saúde no envelhecimento está estreitamente interligado às condições estruturais, sociais, comportamentais e individuais, intervindo no quanto os idosos podem dispor de recursos para alcançar a saúde e a qualidade de vida. Ressalta-se que o conceito de saúde é multidimensional, considerando os aspectos biológicos, físicos, psicológicos, econômicos e sociais, sendo assim, torna-se complexo avaliar se um indivíduo está ou não saudável (BUSATO et al., 2014).

Visto que o envelhecimento implica em um aumento do risco para o desenvolvimento de diferentes tipos de dificuldades, estes aspectos geram a possibilidades de adoecimento e dificuldades de acesso aos recursos protetores disponibilizados na sociedade (RODRIGUES; NERI, 2012). Nesta perspectiva, é notável que atualmente as políticas públicas de resposta ao idoso implementadas, ainda não foram suficientemente capazes de reduzir os problemas associados à vulnerabilidade social deste estrato populacional (KUCHEMANN, 2012).

Neste cenário, observa-se também que a falta de conhecimento sobre o processo de envelhecimento, e a ausência de demonstrações afetivas entre a família e o idoso podem gerar impactos negativos. Desta forma, conhecer as necessidades físicas e emocionais que o indivíduo apresenta, facilitam o planejamento da assistência realizada pelo profissional da saúde, além de agregar qualidade no 
atendimento, promovendo um trabalho satisfatório tanto para a equipe quanto para a população idosa (FERNANDES; SALIMENE, 2015).

Atrelado a estas condições de vulnerabilidade social, podemos citar o abandono. Esta situação não consiste apenas em não ter uma família, ou ser abandonado por esta, mas sim, de uma forma geral, estar desamparado, vivenciando situações estressoras e risco social, ao estar adscrito em um contexto de desproteção de seus direitos fundamentados (KUCHEMANN, 2012). No Brasil, são vários os amparos legais destinados aos idosos os quais se referem à família e a cada membro que integrante seguida pela sociedade e Poder Público. Todavia, observase o abandono familiar de idosos em unidades hospitalares, além da transferência de responsabilidade do Estado para a família (SILVA; MARINHO; DELFINO, 2016).

Neste panorama, é perceptível a presença de dificuldades políticas para acompanhar o acelerado crescimento deste estrato populacional, sendo necessário recuperar as funções e atribuições do Estado, da comunidade e da própria família, aperfeiçoando o apoio necessário ao desenvolvimento de proteção e assistência voltadas aos idosos (SANTOS; SILVA, 2013). Além disso, é importante que haja uma reflexão acerca do cuidado prestado aos idosos pelos profissionais da saúde, sendo necessário reconhecer as necessidades físicas e emocionais que o idoso apresenta, reconhecendo suas especificidades, visto serem indivíduos que requerem um cuidado diferenciado (ALMEIDA; AGUIAR, 2011).

\section{PERFIL CONTEMPORÂNEO DO CUIDADOR DE IDOSOS}

Com o envelhecimento da população brasileira, observa-se uma consequente elevação no número de doenças crônico-degenerativas e aumento do índice de internações hospitalares. Neste sentido, os idosos necessitam de cuidados permanentes, exigindo alterações no estilo de vida e gerenciamento da saúde (SOUZA et al., 2014).

Neste contexto, onde se verifica uma transformação na ordem demográfica de forma brusca e acelerada e sabendo das dificuldades do Estado em não conseguir atender as demandas que são específicas para esta população idosa, o papel do cuidador informal passa a ser primordial (CABRAL; NUNES, 2015). O cuidador informal é o indivíduo que atua de forma voluntária na assistência prestada ao idoso, sem remuneração ou formação profissional específica, sendo que na maioria das vezes é um papel assumido pela mulher, com predominância de esposas e em seguida vêm os descendentes diretos, sendo a filha mais velha a pessoa mais provável para exercer o cuidado, com relação aos demais filhos (HEDLER et al., 2016). Essa modalidade de atenção é configurada como antiga, entretanto, tem se constituído mais explícita com o aumento da expectativa de vida e a reformulação do domicílio como "lócus do cuidado" (COUTO; CASTRO; CALDAS, 2016).

Estudos realizados por Karsch (2003), trazem resultados evidenciando que $59 \%$ das cuidadoras havia idade acima de 50 anos e $41 \%$ com mais de 60 anos. Além disso, 39,3\% das cuidadoras tinham entre 60 e 80 anos e cuidavam de $62,5 \%$ 
de pacientes da mesma faixa etária. Atrelado a isso, é perceptível a presença de sobrecarga do cuidador, relacionando-a ao seu comprometimento funcional e psicológico decorrente da dedicação diária ao cuidado, além das mudanças geradas na vida social, convivência com sintomas depressivos e alterações cognitivas, além da disfunção de papéis ou interrupção da rotina familiar (ROCHA; AVILA; BOCCHI, 2016).

Outro fator que merece destaque é em relação à escolaridade, sendo que estudos demonstram que esta pode influenciar na qualidade da assistência ao idoso, pois é necessária a compreensão de uma gama de situações, tanto na própria assistência como na compreensão do que acontece durante o processo de senilidade (ARAUJO et al., 2013). Desta forma, poder-se-ia dizer, que o cuidador ideal seria aquele com conhecimento em diversas áreas da saúde, dentre elas, as que abrangem o processo de envelhecimento e seus impactos (SAMPAIO et al., 2011).

A partir desta realidade, por meio do Projeto de Lei $\mathrm{n}^{\circ} 4.702$, de 12 de novembro de 2012, foi regulamentada a profissão de cuidador de idosos com o intuito de qualificar a assistência prestada a este estrato populacional. Este Projeto indica a exigência de ensino fundamental e certificado de curso de formação de cuidadores para aqueles que pretendem exercê-lo (DEBERT; OLIVEIRA, 2015). Desta forma, observa-se que o papel do cuidador de idosos transcorre dos conhecimentos básicos reconhecidos na prática diária, sendo necessário o aperfeiçoamento e reflexão acerca do cuidado, para que seja estimulada a sensibilização e empatia pelo outro (FUENTES et al., 2014).

\section{ENSINO TRANSFORMADOR E FORMADOR}

Quando se questiona metodologias de ensino não inferimos somente sobre as técnicas aplicadas em sala de aula, mas ao processo de formação profissional que se estende por diversos momentos experimentados pelos alunos. E conforme verificado com as reflexões atuais sobre o panorama de envelhecimento e o papel do cuidador de idosos, acredita-se que sua construção enquanto profissional perpassa os conhecimentos básicos exigidos pela prática diária, mas à sensibilidade característica de um ser humano que cuida de outro.

Buscando aprofundar as reflexões acerca da formação do cuidador de idosos, acompanhou-se uma turma de alunos do referido curso em uma escola profissionalizante do interior do Rio Grande do Sul, Brasil. A turma era composta por quatro alunos que participaram de uma aula problematizadora, sendo que algumas de suas ideias foram utilizadas para demonstrar o aprofundamento das discussões realizadas, indicando as autorias por meio de codinomes "CI", seguido de números arábicos sorteados aleatoriamente.

Como base para as discussões, dois artigos de opinião sobre a temática abordada no curso foram utilizados, sendo o primeiro intitulado "A necessidade 
de qualidade de vida na terceira idade" ${ }^{\text {, }}$ o qual aborda questões sociais e políticas da contemporaneidade que afetam a qualidade de vida da população idosa. Já o segundo texto, "Novos velhos: o envelhecimento no século XXI"7 traz à tona um apanhado histórico sobre as mudanças que ocorreram no processo de envelhecer e suas repercussões na atualidade.

Os textos foram lidos em voz alta por toda a turma e lançados à discussão pelo professor que se remeteu a exemplos atuais para instigar ainda mais o processo de intervenção aplicado à formação profissional. Após foram lançadas ao grupo quatro questões, norteadas pelos assuntos trabalhados em aula e que serviram para focar a discussão.

A primeira delas consistia em "O que é o envelhecimento?", a partir deste questionamento inicial os alunos remeteram os ideais contemporâneos que focam nas necessidades sociais, traduzindo-se sua essência como um estado vital e passível de modificações conforme o seu contexto, seguem algumas falas:

"O envelhecimento bumano é compreendido como um fenômeno biológico natural, construído socialmente pela própria pessoa ao longo da vida." (CI 3)

"Envelhecer é um processo, isso quer dizer que não podemos escolher começar a envelhecer a partir de uma determinada idade. O envelhecimento começa desde o momento em que nascemos. E ao mesmo tempo a independência e a autonomia também vão mudando à medida que crescemos e envelhecemos." (CI 4)

"Envelhecimento é quando a pessoa vai perdendo as forças geralmente após os 60, 70 anos, esquece onde guardou algum objeto, a comida queima, não tem aquele ânimo todo de se cuidar, sua autoestima vai diminuindo e as dores aumentam." (CI 2)

"O envelhecimento bumano é um fenômeno biológico e natural comum de todo ser vivo."

A apreensão do conhecimento baseia-se primeiramente pela associação entre as experiências vividas no meio pessoal e social, a partir desta constatação atribui-se significados e constantes indagações sobre o assunto (FERNANDES; SALIMENE, 2015). Neste sentido, ao abordar os anseios sociais dos idosos, os alunos inferiram sobre suas próprias experiências, levando em consideração aspectos individuais que passaram a caracterizar-se como coletivos durante a discussão (PETRELLA; PINTO, 2016).

A segunda questão lançada para discussão era "Fale sobre a relação do idoso com a família.". Neste contexto os alunos identificaram a relação existente entre família e respeito, e ainda família e cuidado, ou seja, inconscientemente ligaram o

6 PISSAIA, Luís Felipe. A necessidade de qualidade de vida na terceira idade. Jornal A Hora, Caderno Ideias, Lajeado, mar. 2017.

7 PISSAIA, Luís Felipe. Novos velhos: o envelhecimento no século XXI. Jornal A Hora, Caderno Ideias, Lajeado, fev. 2016. 
bem-estar do idoso com a aceitação do grupo familiar sobre si, conforme alguns relatos a seguir:

'Uma pessoa idosa não é mais como uma pessoa jovem, por isso a família deve ter mais cuidado, dar mais amor e carinho, contar histórias, ficar mais próxima, levar os idosos para passear e se divertir." (CI 4)

"A familia representa para os idosos um fator que influencia significativamente a sua segurança emocional. $O$ ambiente familiar pode determinar as características e o comportamento do idoso." (CI 1)

"Tem família que cuida do idoso com carinho, o idoso se sente útil, ajuda fazer algumas tarefas da casa, conta história pros netos, joga carta, vive feliæ. Outros são roubados pela família e outros não são aposentados, estes são um fardo." (CI 3)

"Tem família que vai cuidar, dar amor, carinho, tem família que vai abandonar, maltratar, vai ver esse idoso como um fardo, algo que não serve mais" (CI 2)

O contexto familiar acalenta a discussão por trazer ao foco as experiências ocorridas em meio aos seus próprios entes e transparecer de diversas maneiras as necessidades afetivas da população idosa (SILVA; MARINHO; DELFINO, 2016). Em diversos estudos publicados nos últimos anos, verificou-se a importância de uma relação saudável entre o idoso e sua família, principalmente em questões emocionais (PETRELLA; PINTO, 2016; SILVA; MARINHO; DELFINO, 2016).

$\mathrm{O}$ terceiro foco de discussão foi permeado pelo questionamento a seguir, "O que é o estatuto do idoso? Comente sobre os direitos trabalhados por ele.". Neste momento, os alunos foram instigados a relembrar as políticas públicas vigentes sobre envelhecimento humano e seus consonantes abordados pela legislação, alguns trechos foram elencados a seguir:

"Garante os direitos assegurados às pessoas com idade igual ou superior a 60 anos. Os direitos são: Saúde, alimentação, transporte, previdência social, habitação, entre outros.” (CI 3)

"Estatuto do idoso foi criado em 2003, é uma lei que foi criada no Brasil, destinada para pessoas acima de 60 anos, e em 2004 esta lei foi ampliada. Direito de moradia, transporte, liberdade, SUS, alimentação, direito de participação na sociedade, etc.” (CI 2)

"É uma Lei. São tratadas questões fundamentais desde garantias prioritárias aos idosos a partir de uma faixa etária dando direitos." (CI 4)

"O estatuto do idoso se propõe uma mudança no comportamento da sociedade aos idosos, como dever da família, da sociedade e do poder público, devem ter direito à vida, à liberdade, ao respeito e à dignidade, direito à saúde, aos alimentos, esportes, laz̧er e do trabalho, previdência e assistência social, habitação e transportes" (CI 1)

As abrangências sociais compõem as discussões de formação do perfil do cuidador de idosos, ao passo que suas condutas são baseadas em princípios regidos por legislações amplamente pensadas e discutidas sobre a realidade da população na terceira idade (MIRANDA; MENDES; SILVA, 2016). Atrelam-se, ainda, as verificações de informações em redes sociais ou mídias de informação diversas 
que abrangem não somente a titulação de determinadas legislações vigentes, mas incorporam sua significância e modo de representá-la (ROSA, 2016).

Para finalizar a discussão, escolheu-se um tema abrangente e que se encontra em evidência por meios das mídias de informação e comunicação, sendo questionado "Quais são os tipos de violência?". Por tratar-se de uma pergunta simples permitiu aos alunos associarem a criatividade perante suas próprias experiências e verificações do meio social ao qual estão inseridas, tratando de diversas formas os meios de violência contra a pessoa idosa, conforme as falas a seguir:

"Violência física, psicológica, negligência e abandono, abuso sexual, autonegligência e abuso econômico." (CI 3)

"Maus tratos, abandono, violência física, violência psicológica, abuso financeiro de material, abuso sexual, negligência e autonegligência." (CI 4)

"Os tipos de violência física são: puxões, queimaduras, beliscões, sedação, amarrar etc."

"Moral, físico, abuso financeiro on material, abuso sexual, negligência, abandono, autonegligência." (CI 2)

As considerações sobre violência representam as próprias atribuições elencadas por meio de mídias vinculadas à população atualmente, a qual delimita sua própria compreensão dos diversos meios e formas de sua realização (ROSA, 2016). A discussão ocupou o tempo de três horas de aula, compondo não somente um meio de teste sobre a formação profissional, mas como a aplicação de uma metodologia diferenciada e em consonância com as necessidades do conteúdo. As repercussões levantadas em questão são enriquecidas a cada aula, fortalecendo ainda mais a aprendizagem dos alunos e transmissão de conhecimento de forma horizontal.

\section{CONSIDERAÇÓES FINAIS}

O envelhecimento humano tornou-se um paradigma social desde o momento em que foi evidenciado em nossa sociedade, devido ao impacto gerado nos determinantes principalmente econômicos e políticos de prestação de serviços. Por meio da reflexão tornou-se visível a inversão da pirâmide etária em evidência na contemporaneidade e que se torna um guia para projeções futuras de aumento do contingente populacional na terceira idade.

Compreenderam-se as modificações no perfil epidemiológico da população idosa e os seus determinantes por meio de um limiar histórico de evolução e modernidade das práticas em saúde que agregaram uma maior expectativa de vida na população mundial. Neste contexto, compreendeu-se o papel desempenhado pelo cuidador de idosos, bem como seu perfil enquanto indivíduo apoiador do processo de envelhecimento, onde atua diretamente nas necessidades que se atrelam às diferentes nuances de suas fases. 
Verificou-se que a profissionalização destes indivíduos torna-se necessário à medida que o contingente populacional idoso aumenta e as demandas por uma assistência qualificada e integral são requisitadas pelos indivíduos. Identificou-se que práticas de ensino baseadas em metodologias de problematização fortalecem a apreensão do conhecimento e posterior contextualização sobre seu papel enquanto profissional.

Tendo-se como base os trechos elencados evidencia-se a amplitude das discussões que ocorrem quando os alunos são instigados para tal situação perante um problema em comum e que cumpra com o entendimento aplicado em sua área de atuação. Compreendeu-se que a discussão de textos atuais aguçam a crítica e reflexão dos alunos, enquanto os questionamentos constroem o cenário de conversação, transformando e reconstruindo os conhecimentos prévios de cada futuro profissional.

Considera-se que a expansão da discussão abarcada pela metodologia exposta neste curso profissionalizante foi capaz de construir novos pensamentos e ideias frente à formação do cuidador de idosos. Instrumentalizou-se a formação reflexiva e integral de um profissional humano e ciente de sua realidade, que atua perante os princípios éticos e que permanece em constante desconstrução e reconstrução do seu saber.

\section{REFERÊNCIAS}

ALMEIDA, Aline Branco Amorim de; AGUIAR, Maria Geralda Gomes. O cuidado do enfermeiro ao idoso hospitalizado: uma abordagem bioética. Revista Bioética, 19 (1): 197- 217, 2011.

ARAUJO, Jeferson Santos; VIDAL, Glenda Marreira; BRITO, Felipe Nunes; GONÇALVES, Débora Cristina de Abreu; LEITE, Djeane Kathe Mascote; DUTRA, Claudia Daniele Tavares; PIRES, Carla Andrea Avelar. Perfil dos cuidadores e as dificuldades enfrentadas no cuidado ao idoso, em Ananindeua, PA. Revista Brasileira de Geriatria e Gerontologia, 16(1): 149-158, 2013.

AZEVEDO, Mônica Cordeiro; GAZETTA, Maria Luísa Barca; SALIMENE, Arlete Camargo de Melo. Envelhecimento e participação social. Acta fisiátrica, 10(3): 102-106, 2016.

BUSATO, Maria Assunta; GALLINA, Luciara Souza; TEO, Carla Rosane Paz Arruda; FERRETI, Fátima; POZZAGNOL, Márcia. Autopercepção de saúde e vulnerabilidade em idosos. Revista Baiana de Saúde Pública, 38(3): 625-635, 2014.

CABRAL, Bárbara Pires de Andrade Lage; NUNES, Ciomara Maria Pérez. Percepções do cuidador familiar sobre o cuidado prestado ao idoso hospitalizado. Revista Terapia Ocupacional Universidade de São Paulo, 26(1): 118-27, 2015.

CAETANO, Ana Cláudia Moura; TAVARES, Darlene Mara dos Santos. Unidade de Atenção ao Idoso: atividades, mudanças no cotidiano e sugestões. Revista Eletrônica de Enfermagem, 10(3): 2017. 
COUTO, Alcimar Marcelo do; CASTRO, Edna Aparecida Barbosa de; CALDAS, Célia Pereira. Vivências de ser cuidador familiar de idosos dependentes no ambiente domiciliar. Revista Rene, 17(1): 76-85, 2016.

DEBERT, Guita Grin; OLIVEIRA, Amanda Marques de. A profissionalização da atividade de cuidar de idosos no Brasil. Revista Brasileira de Ciência Política, 1(18): 7-41, 2015.

FERNANDES, Mary Ellen Bento; SALIMENE, Arlete Camargo de Mello. Cuidando de idosos dependentes no âmbito domiciliar. Revista Portal de Divulgação, 45(5): 2015.

FUENTES, Sônia Azevedo Menezes Prata Silva; FIGUEIREDO, Daniela; MERCADANTE, Elisabeth Frohlich; LODOVICI, Flamínia Manzano Moreira; CERVENY, Ceneide Maria de Oliveira. A importância da capacitação e formação de pessoas que trabalham com idosos em Centros-Dia. Revista Kairós Gerontologia, 17(3): 233-251, 2014.

HEDLER, Helga Cristina; SANTOS, Marlene de Jesus Silva; FALEIROS, Vicente de Paula; ALMEIDA, Maria Aparecida de Araújo. Representação social do cuidado e do cuidador familiar do idoso. Revista Katál., Florianópolis, 19(1): 143-153, 2016.

KARSCH, Ursula. Idosos dependentes: famílias e cuidadores. Cadernos de Saúde Pública, Rio de Janeiro, 19(3): 861-866, 2003.

KUCHEMANN, Berlindes Astrid. Envelhecimento populacional, cuidado e cidadania: velhos dilemas e novos desafios. Sociedade e Estado, 27(1): 165-180, 2012.

LIMA, Alisson Padilha; DELGADO, Evaldo Inácio. A melhor idade do Brasil: aspectos biopsicossociais decorrentes do processo de envelhecimento. ACTA Brasileira do Movimento Humano, 1(2): 2017.

MIRANDA, Gabriella Morais Duarte; MENDES, Antonio da Cruz Gouveia; SILVA, Ana Lucia Andrade da. O envelhecimento populacional brasileiro: desafios e consequências sociais atuais e futuras. Revista Brasileira de Geriatria e Gerontologia, 19(3): 2016.

PETRELLA, Simone; PINTO, Manuel; PEREIRA, Sara. O Idoso e a Educação para os Media: Novos Desafios entre Envelhecimento e Exclusão Social. SOPCOM: Associação Portuguesa de Ciências da Comunicação, 150-155, 2016.

ROCHA, Suelen Alves; AVILA, Marla Andréia Garcia de; BOCCHI, Silvia Cristina Mangini. Influência do cuidador informal na reabilitação do idoso em pós-operatório de fratura de fêmur proximal. Revista Gaúcha de Enfermagem, 37(1): 2016.

RODRIGUES, Natália Oliveira; NERI, Anita Liberalesso. Vulnerabilidade social, individual e programática em idosos da comunidade: dados do estudo FIBRA, Campinas, SP, Brasil. Ciência e Saúde Coletiva, 17(8): 2129-2139, 2012.

ROSA, Maria João Valente. O envelhecimento da sociedade portuguesa. Fundação Francisco Manuel dos Santos, 2016. 
SALMAZO-SILVA, Henrique; LIMA-SILVA, Thais Bento; BARROS, Thabata Cruz de; OLIVEIRA, Eduardo Moreira de; ORDONEZ, Tiago Nascimento; CARVALHO, Gabriela; ALMEIDA, Evany Bettine de. Vulnerabilidade na velhice: definição e intervenções no campo da Gerontologia. Revista Temática Kairós Gerontologia,15(6): 97-116, 2012.

SAMPAIO, Aline Melo Oliveira; RODRIGUES, Fernanda Nunes; PEREIRA, Valquiria Gonçalves; RODRIGUES, Suely Maria; DIAS, Carlos Alberto. Cuidadores de idosos: percepção sobre o envelhecimento e sua influência sobre o ato de cuidar. Estudos e Pesquisas em Psicologia, 11(2): 2011.

SANTOS, Nayane Formiga dos; SILVA, Maria do Rosário de Fátima e. As políticas públicas voltadas ao idoso: melhoria da qualidade de vida ou reprivatização da velhice. Revista FSA, Teresina, 10(2): 358-371, 2013.

SILVA, Andressa Karoline Martins da; MARINHO, Tanimar Pereira Coelho; DELFINO, Maria de Fátima do Nascimento Silva. Abandono familiar de pacientes idosos: uma demanda para o/a assistente social no hospital de urgências de Goiânia. Revista Ciência Escola Estadual Saúde Pública Cândido Santiago-RESAP, 2(1): 1-12, 2016.

SOUZA, Isabela Cata-Preta; SILVA, Ariane Guilherme; QUIRINO, Aurea Cristina dos Santos; NEVES, Michelle Silva; MOREIRA, Luzimar Rangel. Perfil de pacientes dependentes hospitalizados e cuidadores familiares: conhecimento e preparo para as práticas do cuidado domiciliar. Revista Mineira de Enfermagem, 18(1): 2014. 